is viewed by a powerful confocal microscope. Remarkably, as the colour of the incident light is changed from green to red (increasing its wavelength), the area of the chain where the light is confined, as shown by the maximum amount of scattered light, flips from the front end to the back end, in a sharp transition at around $675 \mathrm{~nm}$ (Fig. 1c).

This extreme sensitivity of the light confinement to the incident colour points to wavelength-dependent interference effects, possibly enhanced by delayed interactions of the light with more distant parts of the nanoparticle array. Indeed, nice agreement with the measured switching is obtained in a full dynamic calculation that includes such retardation effects. The authors stress that a quasistatic approach - one that takes into account interactions only in a particle's near field - produces no asymmetric light localization.

Efficient funnelling of the light to a localized spot therefore requires proper tailoring of the interferences caused by coupling between several resonant plasmonic particles. Moreover, the fact that the widely used quasistatic approach is invalid is a severe caveat concerning the reliability of many near-field calculations on plasmonic nanostructures. In this context, it should be noted that a scattering image such as that viewed by de Waele et al. is dominated by areas where the plasmon is converted into emitted light, and it would be interesting to compare this image with a map of the plasmon mode in its near field.

As a final point, it is interesting to return to the comparison of the nanoparticle array to the traditional dipole-array (Yagi Uda) antenna (Fig. 1a) used to pick up radio or television signals from a station in a specific direction. The spacing between the elements in such an antenna is optimized for constructive interference to enhance the directional sensitivity and concentrate the response at the final element - in full analogy to de Waele and colleagues' optical array ${ }^{1}$. Yet there is a significant difference: the nanoparticle antenna relies on electron-plasma oscillations that can be tuned and exploited independently. Moreover, the optical antenna is resonant at wavelengths similar to those of the optical transitions of molecules and quantum dots. One can expect that this resonant coupling will soon be exploited to produce ultra-small, wavelengthsensitive directional sensors or emitters based on nanoparticle chains. In this dynamic field, stay tuned for new surprises soon.

Niek F. van Hulst is at ICFO - The Institute of Photonic Sciences, Mediterranean Technology

Park, Avinguda del Canal Olímpic, 08860

Castelldefels (Barcelona), and ICREA - Institució

Catalana de Recerca i Estudis Avançats, 08015

Barcelona, Spain.

e-mail: niek.vanhulst@icfo.es

1. de Waele, R., Koenderink, A. F. \& Polman, A. Nano Lett. 7, doi:10.1021/nl070807q (2007)

2. Mühlschlegel, P., Eisler, H.-J., Martin, O. J. F., Hecht, B. \& Pohl, D. W. Science 308, 1607-1609 (2005).

3. Taminiau, T. H. et al. Nano Lett. 7, 28-33 (2007).

4. Novotny, N. Phys. Rev. Lett. 98, 266802 (2007).

5. Imura, K., Okamoto, H., Hossain, M. K. \& Kitajima, M. Nano Lett. 6, 2173-2176 (2006).

6. Aeschlimann, M. et al. Nature 446, 301-304 (2007).

7. Krenn, J. R. et al. Phys. Rev. Lett. 82, 2590-2593 (1999).

\title{
GENE TRANSCRIPTION
}

\section{Extending the message}

\section{Patrick Cramer}

\section{During transcription, RNA polymerase catalyses the addition of nucleotides to the growing RNA chain. High-resolution structural snapshots indicate that the polymerase first identifies its substrate, and then incorporates it.}

Life is chemistry - well, at least it is to the molecular biologist. But chemical details remain unclear even for some of the fundamental cellular processes such as gene transcription. In two papers published on pages 157 and 163 of this issue, however, Vassylyev and colleagues ${ }^{1,2}$ provide detailed structural insights into the process of transcription and suggest a two-step mechanism for adding building blocks to a growing RNA chain.

In all living cells, gene transcription is the first step in the decoding of genetic information. During transcription, the enzyme RNA polymerase (RNAP) moves along a DNA template and synthesizes a complementary chain of ribonucleotides - the messenger RNA. Extension of the RNA chain begins with the binding of a nucleoside triphosphate (NTP) substrate to the transcription elongation complex, which consists of RNAP, DNA and RNA. Catalytic addition of the nucleotide to the growing end of the RNA chain then releases a pyrophosphate ion. Finally, translocation of DNA and RNA over the RNAP surface frees the substrate-binding site for the next NTP.

In previous attempts to understand the mechanism of transcription, structural information on the elongation complexes containing eukaryotic RNAPII or bacterial RNAP was obtained ${ }^{3-7}$. These studies showed that DNA enters a cleft-like channel in RNAP. A short hybrid duplex then forms between the DNA template strand and the RNA product above the active site of RNAP at the floor of the cleft. These studies had also indicated possible mechanisms both for the separation of the two DNA strands close to the active site and for RNA-DNA separation at the end of the hybrid.

In some elongation-complex structures, NTP-binding sites were also observed within RNAPII (refs 4-6). The NTP substrate was found to be trapped in the insertion site of RNAP $^{4,6}$ - which is apparently occupied during the catalytic extension of the RNA chain - but also in an overlapping position that slightly differed, indicating the existence of a catalytically inactive NTP-bound state ${ }^{5}$.
8. Maier, S. A. et al. Nature Mater. 2, 229-232 (2003).

9. Sonnichsen, C., Reinhard, B. M., Liphardt, J. \& Alivisatos, A. P. Nature Biotechnol. 23, 741-745 (2005).

10. Ghenuche, P., Cormack, I. G., Badenes, G., Loza-Alvarez, P. \& Quidant, R. Appl. Phys. Lett. 90, 041109 (2007).

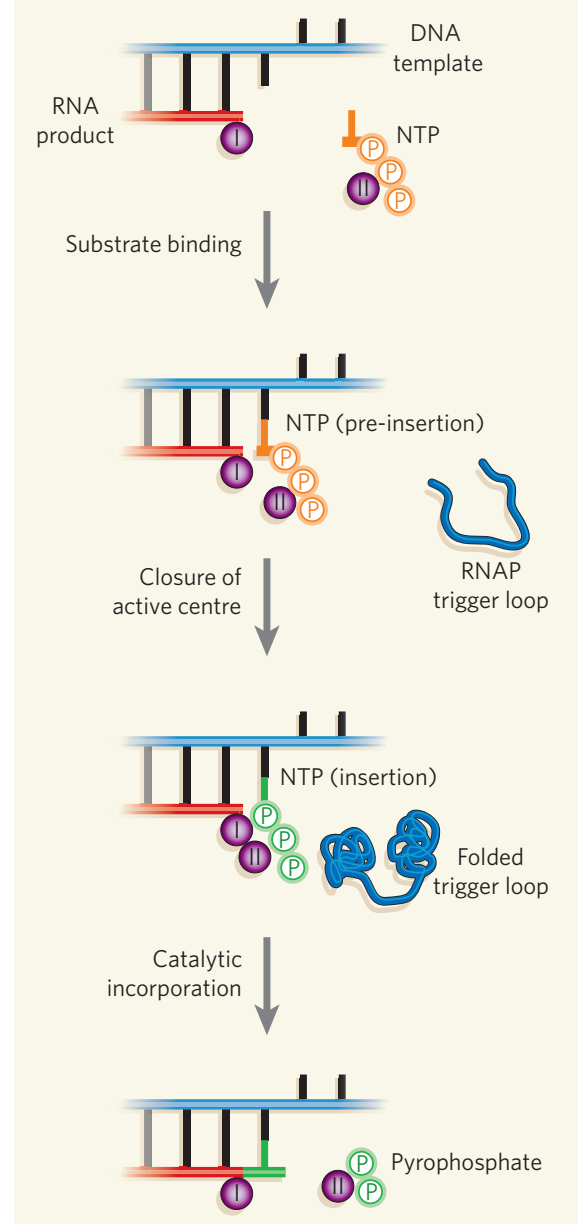

Figure 1 | Two-step mechanism of RNA chain elongation during transcription. Vassylyev et al. ${ }^{1,2}$ found that, during transcription from a DNA template, elongation of an RNA sequence involves the binding of a nucleoside triphosphate (NTP) to the RNA polymerase (RNAP) enzyme in an inactive 'pre-insertion' state; this is characterized by partial folding of the RNAP trigger loop. Complete folding of the trigger loop brings the two metal ions, I and II, of RNAP close together, delivering the NTP to the insertion site, where catalysis leads to nucleotide incorporation and the release of pyrophosphate. 
However, the earlier studies suffered from relatively low resolutions, ranging from 3.5 to $4.5 \AA$.

In one of their two papers ${ }^{1}$, Vassylyev et al. now present the crystal structure of an elongation complex containing bacterial RNAP at $2.5 \AA$ - the highest-resolution structure of an elongation complex for a cellular RNAP, and the first crystal structure of an elongation complex containing bacterial RNAP. They find that the arrangement of DNA and RNA in the complex is very similar to that of RNAPIIcontaining elongation complexes in higher organisms; this reflects a conserved mechanism of transcriptional elongation throughout evolution.

The authors also imaged an RNA single strand within an exit tunnel that leads to the enzyme surface. The exit tunnel could widen and accommodate an RNA duplex, which forms a hairpin structure during pausing and termination of transcription. The elongation complex structure further shows that DNA strands are separated just before the active site, allowing only one NTP to bind at a time.

The new structure of the bacterial elongation complex allowed Vassylyev and colleagues to carry out detailed analysis of the mechanism of nucleotide incorporation into the growing RNA chain in a separate study ${ }^{2}$. They determined two crystal structures of the bacterial elongation complex bound to a non-reactive NTP analogue at a resolution of $3.0 \AA$ - one in the absence and one in the presence of the antibiotic streptolydigin.

In the absence of streptolydigin, NTP binds to two catalytic metal ions at the insertion site, in agreement both with structural and functional information for RNAPII (refs $4,8,9$ ) and with a general two-metal-ion mechanism of action for polymerases ${ }^{10}$. Metal ion I (or A) is persistently bound to RNAP, whereas metal ion II (or B) binds the triphosphate moiety of NTP, and is recruited ad hoc. When NTP binds to the insertion site, a mobile part of the active centre called the trigger loop folds completely ${ }^{2,6}$.

By contrast, in the presence of streptolydigin, the trigger loop folds only partially, and the NTP binds to RNAP in a catalytically inactive 'pre-insertion' state. In this state, the base and sugar groups of NTP are essentially bound as they normally bind during insertion, but its triphosphate group and metal ion II are too far from metal I to allow catalysis. Thus, the antibiotic apparently inhibits transcription by preventing complete folding of the trigger loop, thereby impairing NTP delivery to the insertion site.

The two overlapping but chemically distinct NTP-binding sites in the RNAP active centre support a two-step mechanism of nucleotide incorporation into the growing RNA chain (Fig. 1). The NTP would first bind to an openconformation active centre in an inactive pre-insertion state. Complete folding of the trigger loop then leads to the closure of the active centre, delivery of the NTP to the insertion site, and the formation of all contacts required for catalysis. Biochemical data strongly support the existence of the pre-insertion state, as mutations that interfere with folding of the trigger loop hardly affect NTP binding, but they slow catalysis, apparently because NTP delivery to the insertion site is impaired ${ }^{2}$.

The two-step mechanism helps explain transcription fidelity. Selection of the correct NTP would begin by sampling of substrates in the pre-insertion state, when Watson-Crick base pairing between the correct NTP and the DNA template is established, and RNAP contacts discriminate between an NTP and a deoxy-NTP, to prevent DNA synthesis. The appropriate NTP is then delivered to the insertion site, where its correct base pairing with the template is double-checked by a tight fit in the complementary closed active centre. Subsequent catalysis leads to RNA extension and pyrophosphate formation. It could be the release of pyrophosphate that destabilizes the closed conformation of the active centre ${ }^{11}$, leading to the unfolding of the trigger loop, and enabling translocation of the nucleic acids.

How exactly translocation occurs, however, remains an open question. Another outstanding puzzle is how various external factors that regulate transcription influence RNA synthesis. By mutating specific amino-acid residues in RNAP, researchers should now be able to further define the transcription mechanism and the functions of the trigger loop and other components of this enzyme. The results of Vassylyev and colleagues ${ }^{1,2}$ provide new clues on how to design such mutations and how to address the remaining questions.

Patrick Cramer is at the Gene Center Munich,

Department of Chemistry and Biochemistry,

Ludwig-Maximilians-Universität München,

Feodor-Lynen-Straße 25, 81377 München,

Germany.

e-mail: cramer@Imb.uni-muenchen.de

1. Vassylyev, D. G., Vassylyeva, M. N., Perederina, A., Tahirov, T. H. \& Artsimovitch, I. Nature 448, 157-162 (2007).

2. Vassylyev, D. G. et al. Nature 448, 163-168 (2007).

3. Gnatt, A. L., Cramer, P., Fu, J., Bushnell, D. A. \& Kornberg, R. D. Science 292, 1876-1882 (2001).

4. Westover, K. D., Bushnell, D. A. \& Kornberg, R. D. Cell 119, 481-489 (2004).

5. Kettenberger, H., Armache, K.-J. \& Cramer, P. Mol. Cell 16, 955-965 (2004)

6. Wang, D., Bushnell, D. A., Westover, K. D., Kaplan, C. D. \& Kornberg, R. D. Cell 127, 941-954 (2006)

7. Korzheva, N. et al. Science 289, 619-625 (2000)

8. Sosunov, V. et al. EMBO J. 22, 2234-2244 (2003).

9. Cramer, P., Bushnell, D. A. \& Kornberg, R. D. Science 292, 1863-1876 (2001).

10. Steitz, T. A. Nature 391, 231-232 (1998).

11. Yin, Y. W. \& Steitz, T. A. Cell 116, 393-404 (2004)

\title{
EXTRASOLAR PLANETS
}

\section{Water on distant worlds}

\author{
Heather A. Knutson
}

\section{Is the presence of water a feature common to all gas-giant planets? The first convincing detection of water vapour in the atmosphere of such a planet from outside our Solar System indicates that the answer is yes.}

Gas-giant planets - Jupiter, Saturn, Uranus and Neptune in our own Solar System generally form at large distances from their parent star. Here, radiation is less intense, and so water and other low-mass elements and compounds, such as methane, can be more easily accreted in the form of ices onto the newly formed protoplanet. This process explains why the Solar System's gas giants contain significantly larger quantities of water than does the relatively rocky planet we call home. The rule is expected to hold for gas giants outside our Solar System, which we presume formed through similar processes. Until recently, however, the only planets we were able to study in the necessary detail were those in our own backyard.

On page 169 of this issue, Tinetti and colleagues ${ }^{1}$ provide the first convincing evidence for water vapour in the atmosphere of an extrasolar planet. The hot gas-giant planet, or 'hot Jupiter', that they studied, known as HD 189733 b, is too far away to be imaged directly. Instead, the authors took advantage of the unusual geometry of the system, which is oriented such that the planet eclipses its parent star once every orbit. This transiting geometry of HD $189733 \mathrm{~b}$ was exploited most recently to produce the first-ever infrared 'map' of the temperatures in an extrasolar planet's atmosphere ${ }^{2,3}$. Because, unlike the giants of our Solar System, HD 189733b orbits extremely close to its parent star - at less than 3\% of the Earth-Sun distance - these temperatures range from a toasty 1,200 kelvin on the dayside of the planet to a relatively balmy 970 kelvin on the nightside.

Tinetti et al. used NASA's powerful Spitzer Space Telescope to look for the signal of water absorption in starlight transmitted through the edges of the planet's atmosphere during transit. This method was applied successfully a few years ago to detect sodium in the atmosphere of a hot Jupiter ${ }^{4}$. The authors find that the effective light-blocking area of HD 189733b is slightly larger when measured at a wavelength of $5.8 \mu \mathrm{m}$ than at $3.6 \mu \mathrm{m}$. This difference, they conclude, is the effect of water vapour in the planet's atmosphere, which will absorb more 